University of Nebraska - Lincoln

DigitalCommons@University of Nebraska - Lincoln

2012

\title{
The hardness and strength of metal tribofilms: An apparent contradiction between nanoindentation and pillar compression
}

Corbett C. Battaile

Sandia National Laboratories, Albuquerque, NM

Brad L. Boyce

Sandia National Laboratories, Albuquerque, NM

Christopher Weinberger

Sandia National Laboratories, Albuquerque, NM

Somuri Prasad

Sandia National Laboratories, Albuquerque, NM

Joseph R. Michael

Sandia National Laboratories, Albuquerque, NM

See next page for additional authors

Follow this and additional works at: https://digitalcommons.unl.edu/usdoepub

Part of the Bioresource and Agricultural Engineering Commons

Battaile, Corbett C.; Boyce, Brad L.; Weinberger, Christopher; Prasad, Somuri; Michael, Joseph R.; and Clark, Blythe G., "The hardness and strength of metal tribofilms: An apparent contradiction between nanoindentation and pillar compression" (2012). US Department of Energy Publications. 104.

https://digitalcommons.unl.edu/usdoepub/104

This Article is brought to you for free and open access by the U.S. Department of Energy at DigitalCommons@University of Nebraska - Lincoln. It has been accepted for inclusion in US Department of Energy Publications by an authorized administrator of DigitalCommons@University of Nebraska - Lincoln. 


\section{Authors}

Corbett C. Battaile, Brad L. Boyce, Christopher Weinberger, Somuri Prasad, Joseph R. Michael, and Blythe G. Clark 


\title{
The hardness and strength of metal tribofilms: An apparent contradiction between nanoindentation and pillar compression
}

\author{
Corbett C. Battaile*, Brad L. Boyce, Christopher R. Weinberger, Somuri V. Prasad, \\ Joseph R. Michael, Blythe G. Clark \\ Sandia National Laboratories, P.O. Box 5800, Albuquerque, NM 87185-0889, USA
}

Received 7 September 2011; received in revised form 29 November 2011; accepted 29 November 2011

Available online 1 February 2012

\begin{abstract}
After sliding contact of a hard spherical counterface on a metal surface, the resulting wear scar possesses a complex microstructure consisting of dislocations, dislocation cells, ultrafine or nanocrystalline grains, and material that has undergone dynamic recovery. There remains a controversy as to the mechanical properties of the tribolayer formed in this wear scar. To investigate the properties of this thin layer of damaged material in single crystal nickel, we employed two complementary techniques: pillar compression and nanoindentation. In both techniques, the tests were tailored to characterize the near surface properties associated with the top $500 \mathrm{~nm}$ of material, where the wear-induced damage was most extensive. Pillar compression indicated that the worn material was substantially softer than neighboring unworn base metal. However, nanoindentation showed that the wear track was substantially harder than the base metal. These apparently contradictory results are explained on the basis of source limited deformation. The worn pillars are softer than unworn pillars due to a pre-straining effect: undefected pillars are nearly free of dislocations, whereas worn pillars have pre-existing dislocations built in. Nanoindentation in worn material behaves harder than unworn single crystal nickel due to source length reduction from the fine-grained wear structure.
\end{abstract}

(C) 2011 Acta Materialia Inc. Published by Elsevier Ltd. All rights reserved.

Keywords: Wear; Deformation structure; Dislocations; Compression test; Nanoindentation

\section{Introduction}

Metallic sliding friction is known to produce highly cold worked nanocrystalline or ultrafine-grained layers, via severe plastic deformation, that are confined to the region just below the wear surface [1-5]. Numerous studies have examined the evolution of these subsurface layers, including the formation of steep dislocation gradients and cell walls, as well as processes such as grain refinement and dynamic recovery [6]. In some instances, these tribologically induced surface layers can result in an evolution towards low-friction behavior [5]. However, there is a longstanding controversy about whether these near-surface layers are harder or softer than the parent material [7], a controversy

\footnotetext{
* Corresponding author. Tel.: +1 505844 7039; fax: +1 5058449781 .

E-mail address: ccbatta@sandia.gov (C.C. Battaile).
}

which is propagated by the lack of definitive experimental techniques. A study of the local near-surface mechanical properties of these tribolayers would not only assist in the interpretation of the low-friction evolution process, but also bear relevance to the properties of ultrafinegrained and nanocrystalline metals produced by severe plastic deformation.

Nanoindentation and micropillar compression have emerged as two common techniques to interrograte the mechanical behavior of small volumes, and are therefore relevant tools to apply to wear-induced tribolayers. These tools have proved to be particularly useful for examining size-dependent mechanical behavior. The classical example of size-dependent mechanical behavior is the Hall-Petch effect $[8,9]$, which relates the flow strength of a material to its average grain size. The Hall-Petch relationship, which is observed but still lacks strong theoretical 
underpinnings, has been shown to break down as the dislocation free path is constrained to tens of nanometers [9-11]. Another well-established size dependency occurs in the presence of strong strain gradients, such as in nanoindentation, where geometrically necessary dislocations cause a hardening effect in small volumes [12,13]. Even in the absence of grain boundaries and strain gradients, such as micropillar compression experiments of single crystals, size-dependent mechanical response has been observed [14-17]. This last form of size dependency is related to the role of free surfaces and finite volumes on dislocation production, storage and annihilation. While the purpose of the present work is not to examine size-dependent mechanical properties, it is necessary to be aware of these effects when interpreting the observed behavior using both nanoindentation and micropillar compression techniques.

In this paper, we investigate the mechanical properties of near-surface tribolayers created by a wear process on (initially) single crystal nickel. The wear process induces two subsurface features: a forest of dislocations, with a dislocation density gradient decreasing away from the surface and cell/grain boundaries which transition to the parent single crystal away from the surface. Nanoindentation and microcompression tests were performed on both the nanocrystalline wear material and the virgin, single crystal nickel. This provides a unique side-by-side comparison between the mechanical properties of single crystal material and tribologically "damaged" material on a single sample with otherwise nominally identical conditions. The comparison of nanoindentation and microcompression highlight the effects of confinement and strain gradients in small-scale plasticity. Furthermore, the mechanical tests show an apparent contradiction in behavior: nanoindentation suggests the tribolayer is harder than the single crystal, while microcompression tests conclude that it is softer. These findings illustrate that the mechanical behavior of materials at the nanoscale can be sensitive to not only microstructure and specimen size, but also the geometry and constraint of the specimen and the deformed volume.

\section{Wear track production}

Wear tracks were created on a $\left\{\begin{array}{llll}1 & 1 & 0\end{array}\right\}$ surface of a 99.995\% pure single crystal nickel coupon obtained from Acumet Materials Company. The wear tracks and wear measurements were made using a ball-on-disk linear wear tester in unidirectional sliding mode with a $3.175 \mathrm{~mm}$ diameter $\mathrm{Si}_{3} \mathrm{~N}_{4}(\mathrm{Cerbec})$ ball. To reduce the influence of the surrounding environment, the tribometer was housed in a chamber and the tests were conducted in dry nitrogen $\left(<1 \% \mathrm{RH},<10 \mathrm{ppm} \mathrm{O}_{2}\right.$, and $\left.<100 \mathrm{ppm} \mathrm{H}_{2} \mathrm{O}\right)$. Normal forces of 0.98 and $0.098 \mathrm{~N}$ were applied by deadweights. A $500 \mathrm{mN}$ Sensotec transducer in the load arm measured the tangential (friction) force over a track distance of approximately $1.6 \mathrm{~mm}$. The sliding speed was $3.7 \mathrm{~mm} \mathrm{~s}^{-1}$.

A matrix of wear conditions was examined but, for brevity, only two wear conditions will be discussed here. While these two wear tracks were produced under markedly different conditions and possess different wear-induced damage states, they both exhibited similar trends in mechanical behavior, which is the emphasis of the present study. The two linear wear tracks of interest were created along two different crystallographic directions: $\left\langle\begin{array}{llll}1 & 1 & 0\end{array}\right)$ and $\left\langle\begin{array}{lll}1 & 1 & 2\end{array}\right\rangle$. The $\left\langle\begin{array}{llll}1 & 1 & 0\end{array}\right\rangle$ wear track was created with $0.98 \mathrm{~N}$ of normal force for 1000 cycles, resulting in a steady-state friction coefficient of $\mu \sim 0.5$; the $\left\langle\begin{array}{lll}1 & 1 & 2\end{array}\right\rangle$ direction track was created with $0.098 \mathrm{~N}$ of normal force for 2000 cycles, resulting in a steady-state friction coefficient of $\mu \sim 0.4$. A scanning electron microscopy (SEM) image of the $\left\langle\begin{array}{lll}1 & 1 & 0\end{array}\right\rangle$ track is shown in Fig. 1a. Note that the micropillars created in the wear track are also visible in Fig. 1a. The coefficient of friction during the wear process for both tracks is shown in Fig. 2.

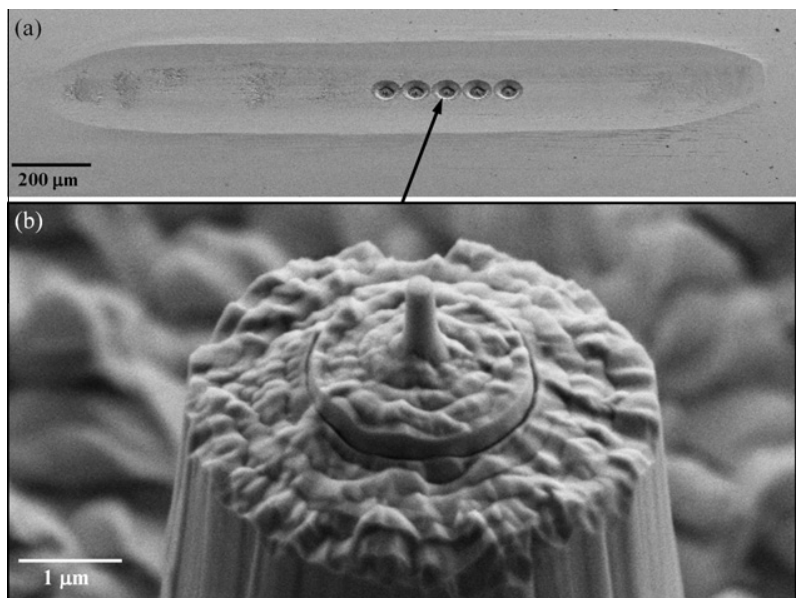

Fig. 1. (a) The wear track left in single crystal nickel showing the creation of five pillars within the track. (b) An example of the as-fabricated submicron pillar.

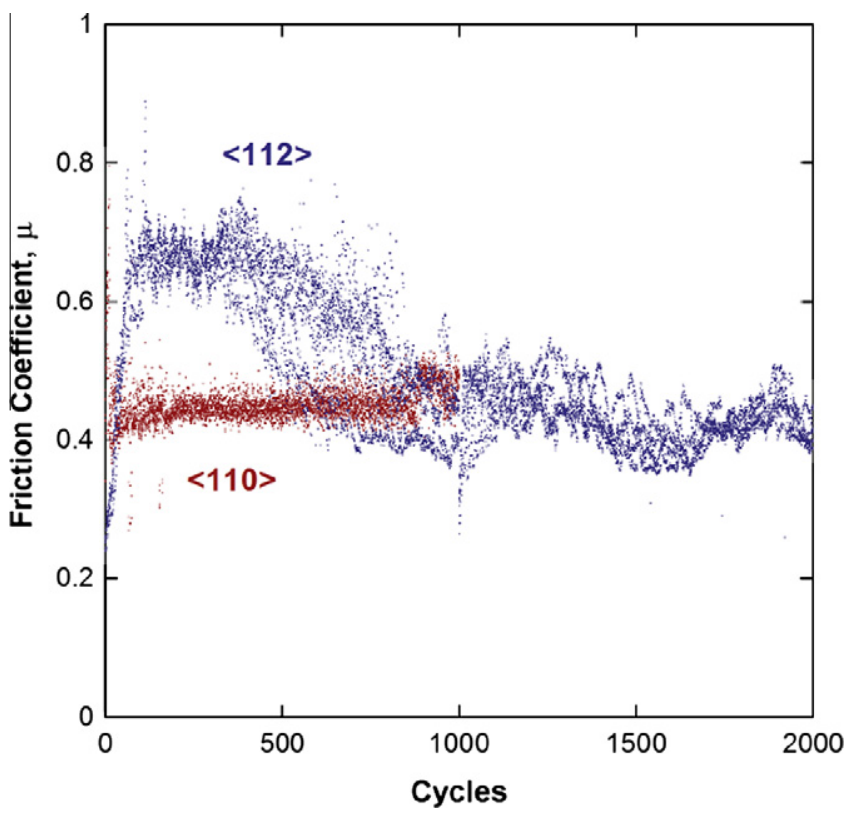

Fig. 2. The coefficient of friction measurements for the two wear tracks. 
The underlying friction-induced defects and grain structures for both wear tracks were examined using cross-sectional focused-ion-beam (FIB) lift-out of transmission electron microscopy (TEM) foils taken at the centerline of the wear track along the wear direction [18]. The bright-field TEM images in Fig. 3 are generally consistent with typical metallic substructures $[6,19,20]$. Wear-induced substructures in metals typically form forest dislocations which organize into brick-shaped "lamellar" dislocation cells elongated along the sliding direction. As wear continues, these cells subsequently evolve into high-angle grain boundaries with dynamic recovery of the intragranular defects. The $\left\langle\begin{array}{llll}1 & 1 & 0\end{array}\right\rangle$ wear track was dominated by partially recovered brick-like grains that were elongated along the sliding direction. The grains were typically 50-300 nm thick and $100-600 \mathrm{~nm}$ long. Our prior work on $\mathrm{Ni}$ wear tracks produced under similar conditions suggest that this substructure consists of a combination of low- and high-angle grain boundaries, with only weak crystallographic texture. The $\left\langle\begin{array}{lll}1 & 1 & 2\end{array}\right\rangle$ wear track also possessed elongated grains or subgrains in the near-surface region. The grain boundary organization only persists to a depth of $\sim 500 \mathrm{~nm}$ in the $\left\langle\begin{array}{lll}1 & 1 & 2\end{array}\right\rangle$ track, whereas the grain boundary network persists to a depth of $5 \mu \mathrm{m}$ in the $\left\langle\begin{array}{llll}1 & 1 & 0\end{array}\right\rangle$ track. Also, the dynamic recovery process appears less complete in the $\left\langle\begin{array}{llll}1 & 1 & 2\end{array}\right\rangle$ track, with many more forest dislocations compared to the $\left\langle\begin{array}{lll}1 & 1 & 0\end{array}\right\rangle$ track. The unworn material was also imaged by TEM for comparison. As expected, the single crystal unworn material contains essentially no substructure, with only a few dislocations present associated with a polishing-induced Beilby layer.

\section{Pillar compression of wear tracks and unworn single crystal nickel}

Samples for compression testing were micromachined into the nickel surface using a dual-platform FIB of gallium $\left(\mathrm{Ga}^{+}\right)$ions. This was accomplished in a multi-step process. First, the areas of interest were located on the sample and covered with a protective platinum layer using consecutive electron- and ion-beam-assisted depositions. This process ensured that the ion beam irradiation did not alter the microstructure in the fine-grained wear scars. Second, pedestal preforms were milled into the surface of the nickel sample. These preforms were $5 \mu \mathrm{m}$ in diameter and approximately $10 \mu \mathrm{m}$ in height, with sufficient material removed from the perimeter to allow access for the compression experiment. Third, a submicron pillar was milled into the top of each preform. The final pillar dimensions were kept as close as possible to a diameter of $250 \mathrm{~nm}$ and a height of $500 \mathrm{~nm}$. The pillars were located as close to the center of the wear scars as possible. After milling, each of the pillars was imaged and measured using a scanning electron microscope. Fig. 1a shows an SEM example of a $\left\langle\begin{array}{llll}1 & 1 & 0\end{array}\right)$ wear track containing five milled pillars and Fig. $1 \mathrm{~b}$ shows a magnified view of one of the pillar-on-pedestal samples.
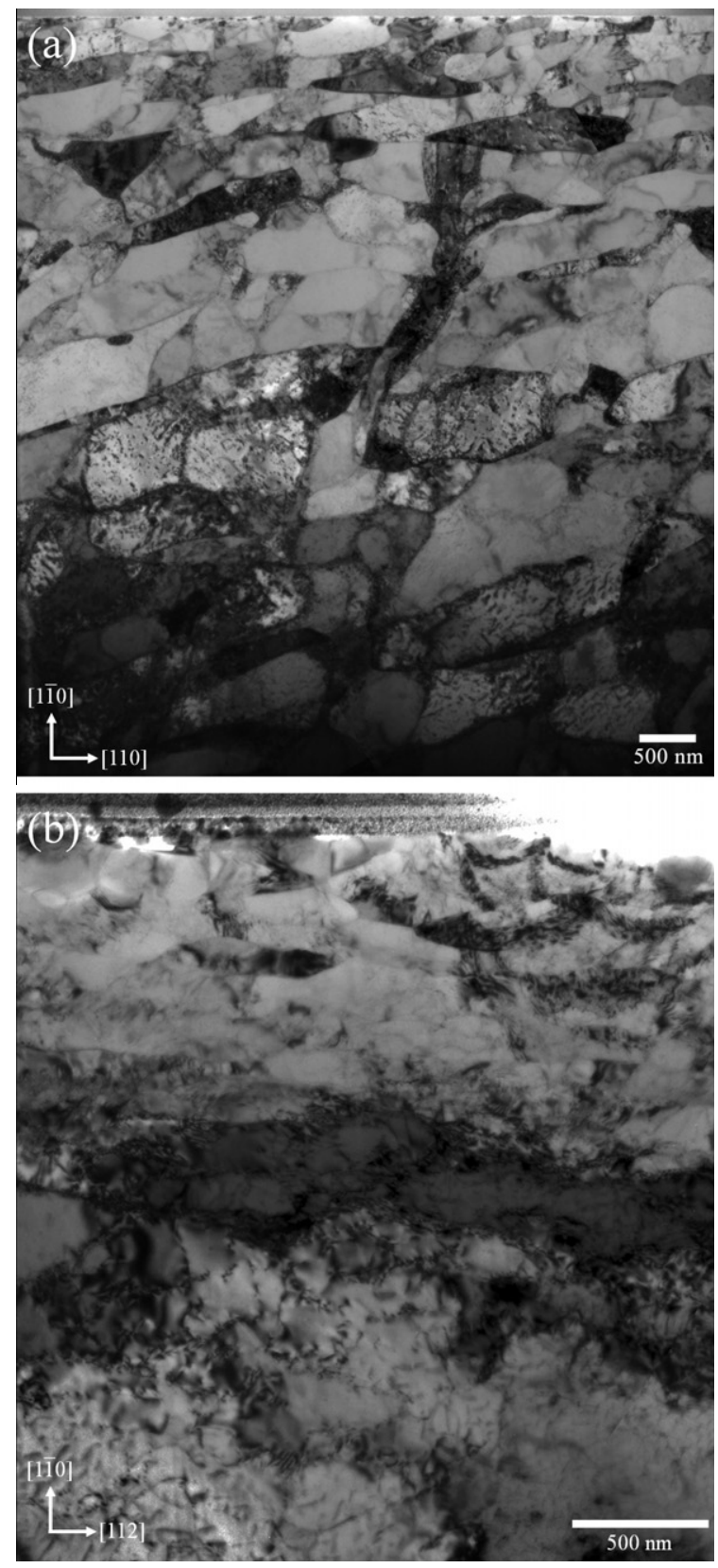

Fig. 3. Bright-field TEM images of the near-surface wear-induced grain boundary and defect content for the (a) $\left\langle\begin{array}{llll}1 & 1 & 0\end{array}\right)$ and (b) $\left\langle\begin{array}{llll}1 & 1 & 2\end{array}\right\rangle$ wear tracks. In both cases, sliding occurred from left to right.

Micropillar compression tests were performed on nine pillars: three from the $\left\langle\begin{array}{llll}1 & 1 & 0\end{array}\right)$ wear track, three from the $\left\langle\begin{array}{llll}1 & 1 & 2\end{array}\right\rangle$ wear track and three from unworn base material as a control group. The tests were performed with a Hysitron Performech TriboIndenter using a $12 \mu \mathrm{m}$ diameter flat punch. The resolution of the TriboIndenter was limited to the noise floor of the instrument, which was measured to be $\pm 0.68 \mathrm{~nm}$ displacement and $\pm 1.29 \mu \mathrm{N}$ force $( \pm 1 \mu \mathrm{N}$ standard deviation). This noise floor was measured while the compression punch was held in contact with the first micropillar immediately prior to testing to best represent the actual test conditions. The compression tests were performed in feedback displacement control at a loading rate 
of $0.5 \mathrm{~nm} \mathrm{~s}^{-1}$ to a total displacement of $100 \mathrm{~nm}$. For pillars that are nominally $500 \mathrm{~nm}$ in height, this corresponds to a strain rate of $0.001 \mathrm{~s}^{-1}$ and a total engineering strain of $\sim 20 \%$.

Because the compression tests each took nearly 4 min to complete, care was taken to eliminate the effects of instrument drift. This was accomplished by allowing the instrument to settle with the compression platen in contact with each sample for no less than $30 \mathrm{~min}$, then collecting drift data prior to each test; applying a linear correction to the acquired data to account for the observed drift; and introducing $10 \mathrm{~s}$ dwell periods at the beginning and peak load of each test to monitor any remnant drift.

Engineering stress-strain curves for the two wear tracks and the unworn material are shown in Fig. 4. Engineering stress was calculated using the measured load and the cross-sectional area corresponding to the median pillar radius. Engineering strain was calculated as the change in length per unit original length, i.e. $\epsilon=\frac{\Delta L}{L}$.

Fig. 4 and Table 1 show that the tribolayer pillars are weaker than a $\langle 110\rangle$ single crystal pillar of the same size. The two different wear tracks show qualitatively similar stress-strain responses. To quantitatively compare strengths between the different pillars, we consider flow strengths measured at two offsets: $5 \%$ and $10 \%$ strains. Evaluating flow stresses at relatively large values of strain is standard practice [21], and is intended to avoid problems arising from misalignment between the compression and pillar axes. The values extracted for three different pillars

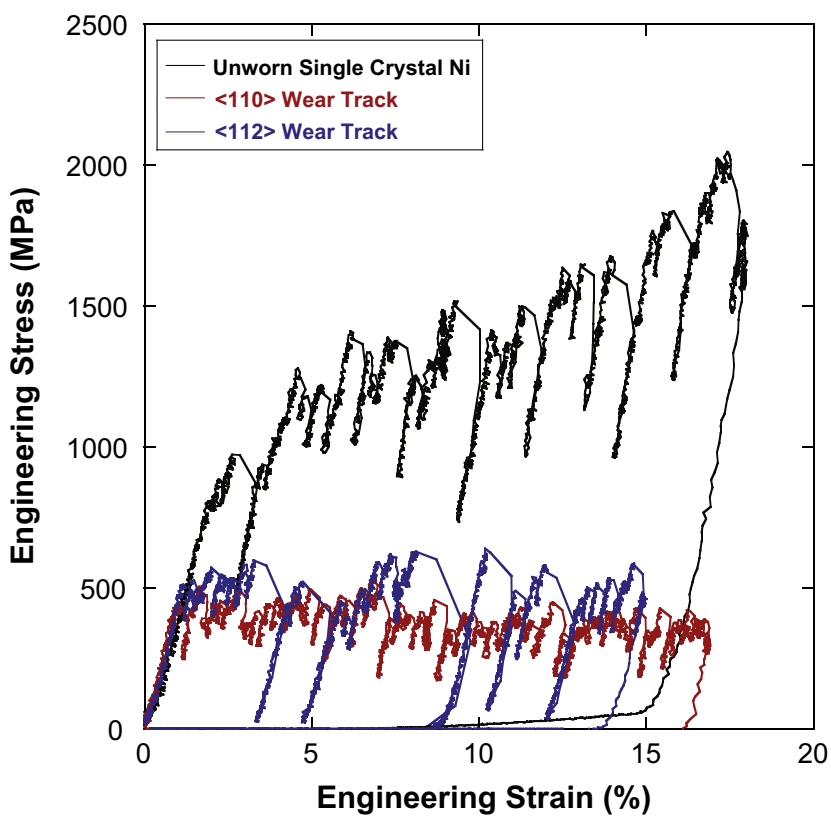

Fig. 4. Engineering stress-strain curves from the two different wear tracks and the unworn single crystal parent material. The $\left\langle\begin{array}{llll}1 & 1 & 0\end{array}\right)$ single crystal exhibits a higher flow stress for the same size pillars and shows strain hardening. The two wear tracks, created in the $\left\langle\begin{array}{llll}1 & 1 & 0\end{array}\right\rangle$ and $\left\langle\begin{array}{llll}1 & 1 & 2\end{array}\right\rangle$ directions, show similar flow stresses and no strain hardening. For each of the three conditions, three pillars were compressed, but only one of each is presented here for the sake of clarity. The other repetitions showed similar behavior, as compiled in Table 1.
Table 1

The strength of all nine pillars, evaluated at 5\% and 10\% flow stresses.

\begin{tabular}{llll}
\hline Condition & Pillar & $5 \%$ Flow stress $(\mathrm{MPa})$ & $10 \%$ Flow stress $(\mathrm{MPa})$ \\
\hline$\left\langle\begin{array}{llll}1 & 1 & 0\end{array}\right\rangle$ & 1 & 350 & 350 \\
& 2 & 800 & 800 \\
& 3 & 750 & 400 \\
$\left\langle\begin{array}{lll}1 & 1 & 2\end{array}\right\rangle$ & 1 & 500 & 600 \\
& 2 & 800 & 850 \\
& 3 & 750 & 750 \\
Unworn & 1 & 900 & 1300 \\
& 2 & 1200 & 1400 \\
& 3 & 1100 & 1200 \\
\hline
\end{tabular}

for each wear condition $\left(\begin{array}{llllll}1 & 1 & 0\end{array},,\left\langle\begin{array}{lll}1 & 1 & 2\end{array}\right\rangle\right.$ and unworn) are listed in Table 1. The unworn pillars are approximately $1 \frac{1}{2}-3$ times stronger than those in the wear tracks.

The strengths of the pillars are not the only features of the stress-strain curves that differ between the worn and unworn pillars. For example, the unworn single crystal pillars exhibit nearly linear strain hardening, with a modulus between 3.5 and 5.5 GPa. By comparison, the wear track pillars show no noticeable strain hardening with virtually constant flow stress for all strains up to $20 \%$. One exception is pillar \#3 in the $\left\langle\begin{array}{lll}1 & 1 & 0\end{array}\right\rangle$ wear track (see Table 1), which exhibits apparent softening between $5 \%$ and $10 \%$ strain.

Post-mortem SEM and cross-sectional TEM images of the pillars were taken to determine the mode of deformation. Corresponding before-and-after images were taken of each micropillar using a Zeiss Supra 55VP field emission scanning electron microscope. Typical in-lens images are shown in Fig. 5. As seen in Fig. 5g-i, the unworn single crystals deform via double slip. This is expected because the $\left\langle\begin{array}{llll}1 & 1 & 0\end{array}\right\rangle$ orientation has two high Schmid factor slip planes and two inactive slip planes. The deformation in the single crystal pillars matches observed deformation patterns in other high symmetry pillars [21] showing generally homogeneous deformation of the pillars and multiple active slip systems.

In contrast, the deformation observed in the wear track pillars is much more localized as shown in Fig. 5a-f. Deformation is largely confined to the top half of the pillar, with little or no discernible deformation occurring in the bottom half. In addition, deformation appears to have occurred either by the formation of a single dominant shear feature (possibly a single active slip plane), as in Fig. 5f, or by the activation of multiple adjacent slip systems, as in Fig. 5 b. These deformation patterns have been observed in single crystal, low symmetry orientations [17], suggesting that the deformation in tribolayer pillars behaves like lowsymmetry single crystal pillars in grains that have high Schmid factors. Nonetheless, while there is a clear and consistent distinction here between images of deformed wear track material and unworn material, there is no significant distinction between the two different types of wear tracks.

This distinction of homogeneous deformation in the unworn pillar and highly localized deformation in the worn pillar belies differences in the underpinning slip process. 

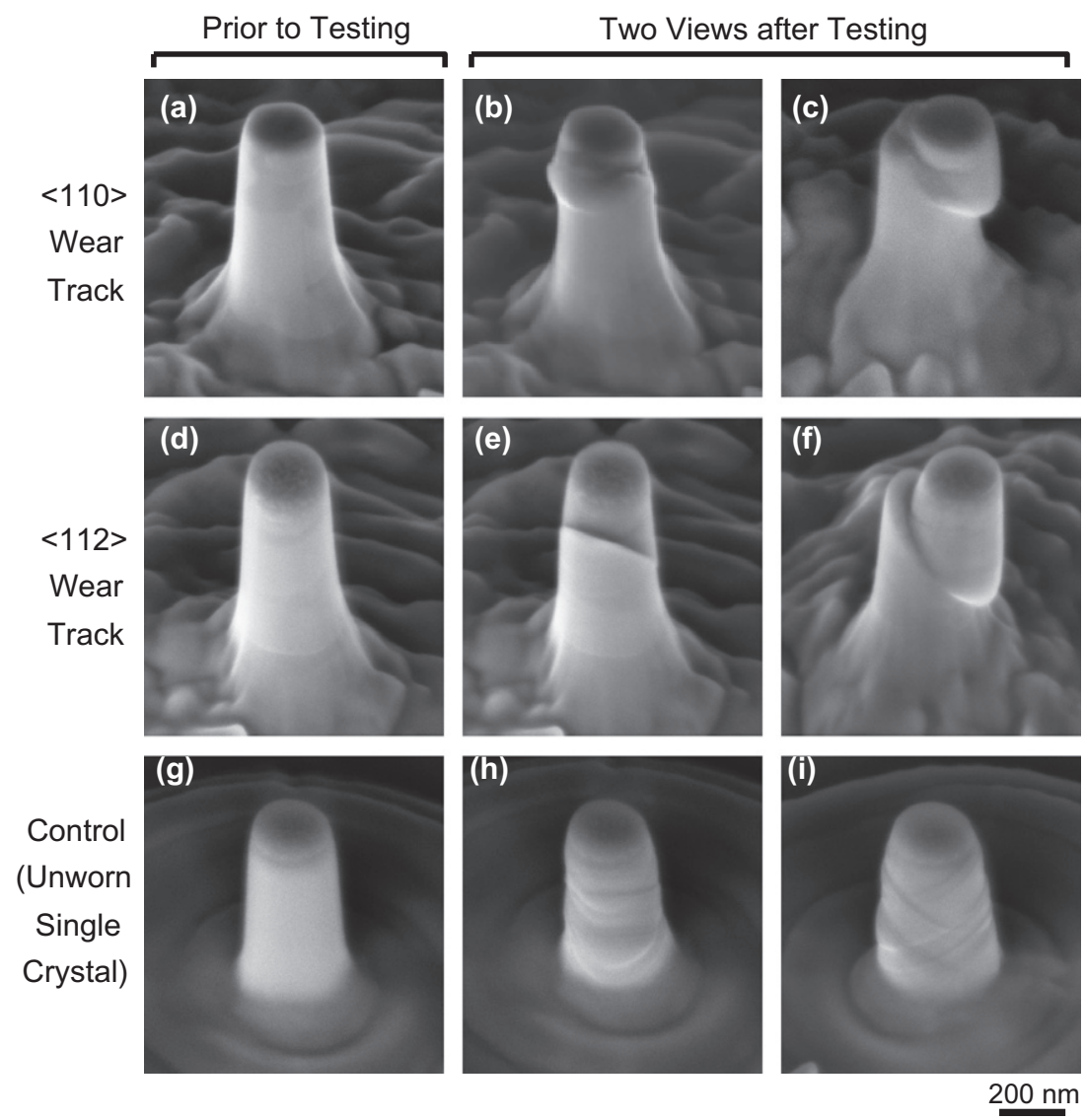

Fig. 5. Before and after SEM images of pillars. (a) $\langle 110\rangle$ wear track pillar before testing and (b and c) SEM images after testing showing localized deformation. (d) $\left\langle\begin{array}{lll}1 & 1 & 2\end{array}\right\rangle$ wear track pillar before testing and (e and f) views after testing also showing localized deformation. (g) $\langle 110\rangle$ single crystal pillar before testing and (h and i) after testing showing homogeneous double slip.

When slip is homogeneous in the unworn single crystal case, this suggests that each individual source is readily depleted or otherwise impeded after a few dislocations have passed, resulting in small surface ledges; only when the stress is increased can a new source be activated. However, in the case of the localized slip in the worn pillar, there is a single dominant source that activates and continues to plow dislocations, creating a single very large ledge with no additional stress needed. These two distinct slip morphologies are entirely consistent with the distinctly different work hardening slopes shown in Fig. 4.

TEM samples of compressed pillars were extracted from each of the wear conditions, using the same procedure outlined above. Fig. 6 shows TEM images from pillars in the two wear tracks. Localized plastic deformation is clearly evident, which, as previously mentioned, does not exist in the single crystal pillars. The cell structures are also clearly evident, showing cell sizes of around a few hundred nanometers. In addition, a significant number of dislocations are visible within the cells. The regions of localized plastic flow do not appear to follow grain boundaries, suggesting the absence of significant grain boundary sliding or related processes. The high dislocation content in these small pillars suggests that the mechanism of deformation is conventional dislocation plasticity.

\section{Nanoindentation of wear tracks and unworn single crystal nickel}

Nanoindentation measurements of the hardness of the three different films were performed using a Hysitron Performech TriboIndenter with a Berkovitch tip with radius less than $45 \mathrm{~nm}$. Nanoindentation hardness was measured as a function of indentation depth ranging from 20 to $240 \mathrm{~nm}$ using the well-established Oliver and Pharr method [22], modified to include multiple partial unloads during each indentation. Fig. 7 shows the absolute hardness for each wear track compared to the unworn single crystal nickel. From these data, it is clear that the tribolayers are harder than the single crystal films by a factor of 2 or more. Using a Tabor factor of 2.9, the flow stresses of the single crystal and tribolayers can be roughly estimated as $600 \mathrm{MPa}$ and 1.5-2.0 GPa, respectively. Caution should be exercised with the quantitative values for flow stress implied by the Tabor relationship, since in this size-affected regime nanoindentation hardness can be elevated by the incorporation of geometrically necessary dislocations [23]. Nevertheless, in this direct comparison of identically sized indents with and without wear damage, the Tabor relationship reinforces the idea that there is a "stronger" response in the wear track material as observed by nanoindentation. 

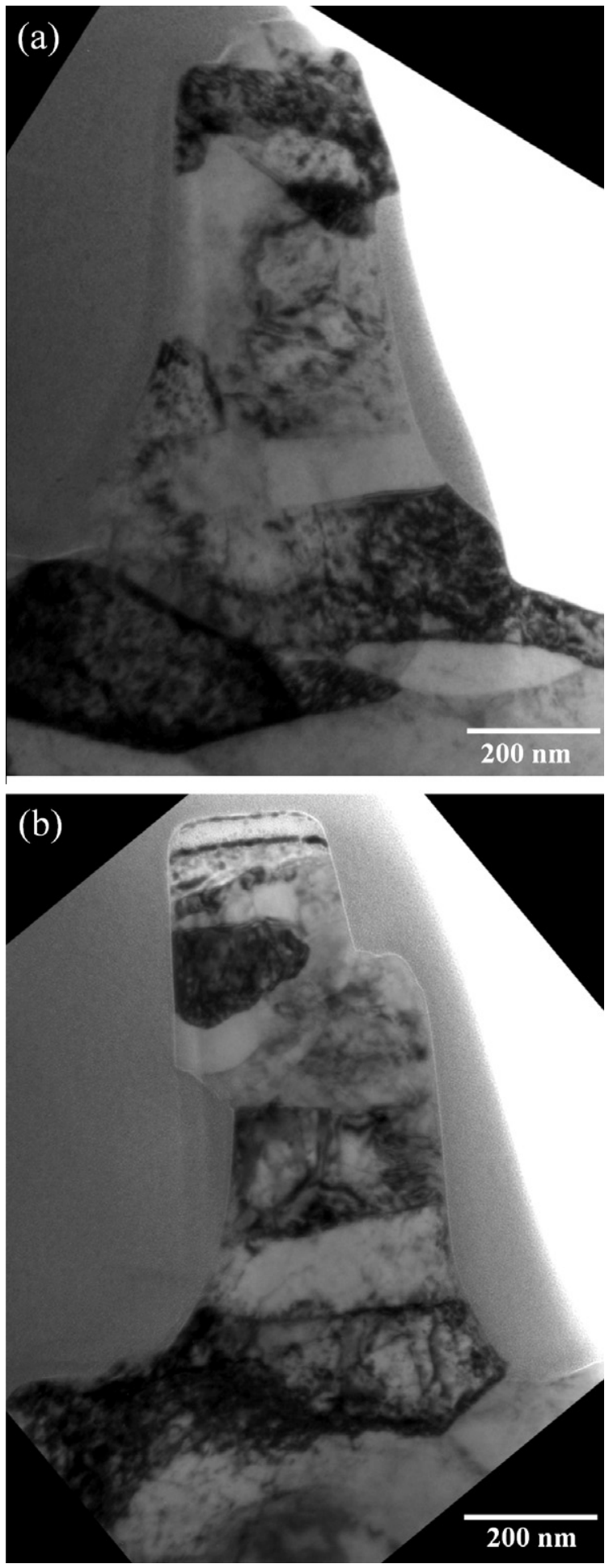

Fig. 6. Bright-field TEM images of deformed pillars from the (a) $\left\langle\begin{array}{lll}1 & 1 & 0\end{array}\right\rangle$ and (b) $\left\langle\begin{array}{llll}1 & 1 & 2\end{array}\right\rangle$ wear scars.

The increased nanoindentation hardness of the tribofilms as compared to the single crystals was expected due to dislocation forest (Taylor) and grain boundary (Hall-Petch) hardening.

In all cases, the nanoindentation behavior in Fig. 7 shows a clear depth dependence: the material is harder at

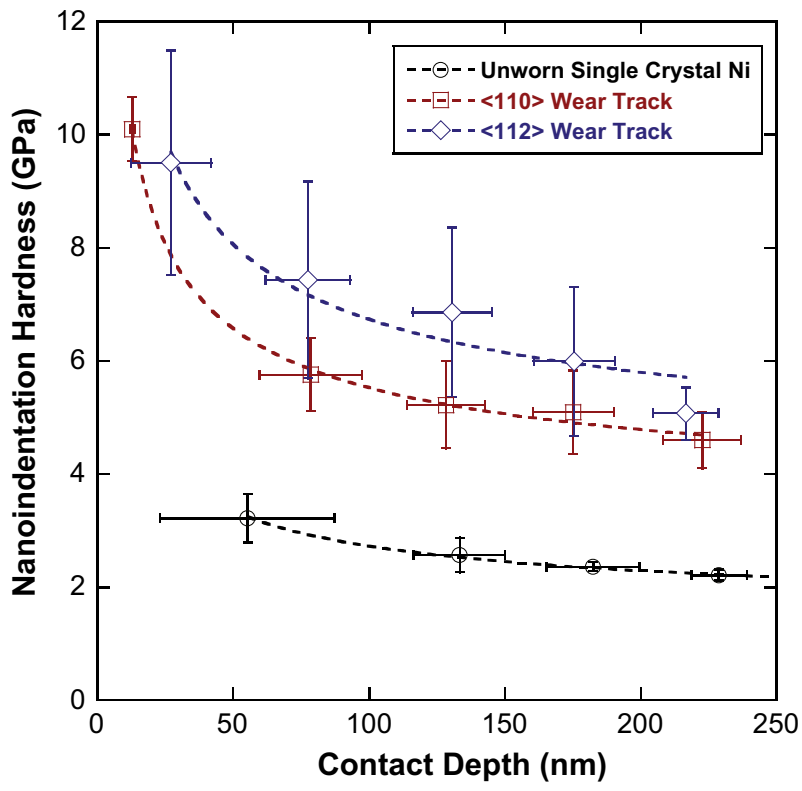

Fig. 7. Hardness data for the single crystal and wear track material. The three dashed lines are curve fits of hardness proportional to contact depth raised to $-1 / 2$ power, consistent with Ref. [12].

shallow depths. Depth-dependent nanoindentation hardness has been studied extensively elsewhere and is not the focus of the present study. Nevertheless, it is worthwhile pointing out that in the present study there are at least two independent sources for this increase in hardness at shallower depths. First is a classic indentation size effect typically attributed to geometrically necessary dislocations in the presence of steep strain gradients [12]. More recently, due to the emergence of similar size-scale dependencies in micropillar compression which cannot be explained by strain gradient concepts, size-dependent hardening has been argued on the basis of a reduction in dislocation sources in small volumes [24,25]. In nanoindentation, the size effect is expected to cause hardness to scale with the reciprocal square root of contact depth $[12,24]$, and such a relationship was employed to fit the data in Fig. 7. A second source of depth-dependent hardness, in addition to the classical indentation size effect, comes from the graded nature of the substructures that form underneath the wear scar. There are gradients in dislocation content and grain/cell size that decay away from the wear surface. This graded substructure would be expected to also contribute to increasing hardness at shallow depths. Note that this second source of depth-dependent hardness does not contribute to the behavior of the unworn single crystal.

\section{Discussion}

\subsection{Deformation mechanism in pillars}

In spite of the small size of the pillars and their even finer grain size, the deformation mode shown in Figs. 5 and 6 suggests conventional dislocation slip-mediated 
plasticity, with no evidence of alternative deformation modes such as twinning or grain boundary sliding. Instead, deformation appears to be due to conventional dislocation plasticity with significant shear localization. In the single crystal parent material, slip steps were present all along the height of the pillar, with at least two slip systems active, as shown in Fig. 5i. However, the tribolayer pillars experienced shear localization, with only a single confined shear zone contributing to a vast majority of the strain. Why do the single crystal pillars deform by slip steps distributed homogeneously throughout the pillar whereas the tribolayer pillars deform by slip localized in a dominant shear band? Since the pillars are of nearly identical size, and produced by the same method, the only distinction is due to the presence of high dislocation densities of order $10^{15} \mathrm{~m}^{-2}$ [1], dislocation cell walls, high-angle grain boundaries and excess free volume. In the undeformed single crystal condition, the entire pillar possesses a nearly homogeneous resistance to source activation and exhaustion. However, in the tribolayer pillar the complex heterogeneous substructure possesses locally weak sites ("hot spots") where deformation is preferred, due to either easy activation or lack of exhaustion. This conjecture might explain the differences in slip morphology, but would require further investigation to be proved conclusively.

\subsection{Apparent contradiction between pillar compression and nanoindentation}

The present study is novel in that both pillar compression and nanoindentation techniques were used on the exact same material to evaluate mechanical performance. The nanoindentation technique was the de facto standard technique for small volume mechanical measurements until the pillar compression technique was introduced in 2004, after which pillar compression has dominated the literature. When considered together, these pillar compression and nanoindentation data indicate that the two testing methods can produce apparently contradictory results. Nanoindentation suggests that the wear scars are about twice as hard as the single crystal. In contrast, pillar compression suggests that the wear scars are considerably softer than the single crystal. While recent efforts have recognized that in micro- and nanoscale testing the observed strength depends on size, it is less obvious - and less well recognized - that the observed strength depends substantially on the testing method.

As previously mentioned, the indentations size effect gives rise to a stronger response at shallower indents. The classic model of strain gradient plasticity theory, however, has been called into question by many recent experiments and simulations [24-28], suggesting that source-limited deformation may be a more likely cause for hardening in submicron indents. Using this interpretation, our results suggest that harder wear track material at these indents is caused by the further reduction in the number and length of glissile sources probed by indentation due to the pre- existing damage substructure of the wear track material. The wear track material can be considered a "pre-strained" material and the hardness trends can be compared against nanoindentation on pre-strained coarse-grained metals $[29,30]$. These materials show that pre-strain generally increases hardness, but only for indentations depths greater than $250 \mathrm{~nm}$. For smaller indents, there is no difference in hardness, supporting the notion that at these depths the hardness and strength are controlled by source limited deformation. Thus, the wear track increase in hardness observed here is likely caused by source-limited or source-truncation hardening rather than conventional Taylor hardening.

This still does not explain why the tribolayer pillars are softer than the single crystal parent material. At the submicron scale, these single crystal pillars are actually quite strong. The strength of conventional macroscale single crystal nickel would be expected to be below $100 \mathrm{MPa}$. The enhanced strengths of the $250 \mathrm{~nm}$ diameter single crystal pillars arise from a well-documented mechanism whereby single crystal pillar strength increases with decreasing pillar size $[17,21,31]$. While the increased strength is thought to be due to a reduction in dislocation sources, the nature of the sources is still debated [32-40]. Two competing models of the nature of the sources, and hence the size effect, are single arm sources [41] (truncated Frank-Read sources) and nucleation from the free surface [42]. Experimental work has also shown that a transition occurs near $100 \mathrm{~nm}$, where a strength plateau is reached, which has been interpreted to be a change from single arm sources to surface nucleation [43]. Recently "prestraining" effects have also been documented in micropillar compression $[44,45]$ : when a pillar is pre-deformed to build in defects, it subsequently is softer than unstrained single crystal pillars. Note that this behavior is opposite to the strain hardening observed at the macroscopic scale.

The tribolayer pillars, similar to the pre-strained single crystal micropillars, are softer than the parent material micropillars. The cause is likely the same: the injection of defects into the pillars via the wear process populates the pillars with mobile dislocations that can contribute to plasticity and reduce the apparent strength of the material. It is worth noting that a similar softening effect was also observed in the $60 \mathrm{~nm}$ grained $\mathrm{Ni}-4.4 \% \mathrm{~W}$ [46]. In that work, the softening effect was attributed to a transition to grain boundary sliding and twinning as the pillar diameter approached the grain size. Such mechanisms were not observed in these experiments, most likely because the presence of a high defect population obviates the need for additional deformation mechanisms.

The explanations of hardening in nanoindentation and pillar compression may still appear contradictory since both use the argument of source-limited deformation. In the pillars, the grain size and pillar size are approximately equal, thus the grains do not provide additional constraints on the source length. However, they do provide additional sources, which enhances the probability of finding weak 
sources and thus reduces the strength. In contrast, in nanoindentation the grain size limits the maximum source length, making the wear tracks stronger.

Care must be taken in comparing the two methods because they probe different aspects of plasticity in confined volumes. For micropillars, the uniaxial nature of the loading tests for the weakest link [35,47], while nanoindentation probes a small volume around the indentation. Furthermore, nanoindentation also includes residual stress that would be removed when pillars are created. The effect of the residual stress is not known exactly, though we expect it to be moderate because the material has already undergone dynamic recovery.

From this set of apparently contradictory data, we are left with the unanswered question of whether the wear track is harder or softer than the parent material. To resolve this contradiction, we have to reflect on why these two nanomechanical test methods produce distinctly different trends: by the nature of these two methods, they probe different volumes of differently prepared material with different boundary conditions/constraints. Clearly, of the two methods, the nanoindentation method more closely mimics the constraint associated with tribological wear, whereas the free surfaces associated with pillar compression create a nonrepresentative boundary condition. Based on this assessment, we can imply that, from the perspective of friction and wear conditions, the tribolayer is more resistant to deformation than the neighboring parent material.

\section{Conclusions}

Heavily dislocated tribofilms were created on the $\left\{\begin{array}{lll}1 & 1 & 0\end{array}\right\}$ surface of pure single crystal nickel by kilocycle wear testing. The mechanical properties of both the parent single crystal and the wear tracks were measured by micropillar compression and nanoindentation. Pillar compression showed the wear track material to be approximately twice as soft as the single crystal, whereas nanoindentation indicated the nanocrystalline material to be about twice as hard as the single crystal. Defects (e.g. grain boundaries and dislocations) generated by wear reduce the strength of the nanocrystalline sub-micron pillars as compared with the single crystal pillars, which are strengthened by dislocation source starvation. The abundance of grain boundaries in the nanoindented material reduces the average source length and hence strengthens the material as compared to the nanoindentated single crystal. These findings indicate that different testing methods can produce qualitatively different measurements of the mechanical properties of nanomaterials, and that care must be taken in the interpretation thereof. The boundary conditions of nanoindentation more closely replicate the constraints associated with tribological wear. This suggests that, from the perspective of friction and wear experiments, the tribolayer is more difficult to deform than the single crystal nickel material.

\section{Acknowledgements}

The authors thank Luke N. Brewer, Thomas E. Buchheit, Thomas W. Scharf, Rand D. Garfield, Alice C. Kilgo, Bonnie B. McKenzie and Michael J. Rye for their contributions to this work. B.L.B. and B.G.C. are supported by the US Department of Energy, Office of Basic Energy Sciences. Sandia is a multiprogram laboratory operated by Sandia Corporation, a Lockheed Martin Company, for the United States Department of Energy's National Nuclear Security Administration under Contract DE-AC04-94AL85000.

\section{References}

[1] Rigney D, Naylor MGS, Divakar R, Ives LK. Mater Sci Eng A 1986;81:409-25.

[2] Rainforth W, Stevens R, Nutting J. Philos Mag A 1992;66:621-41.

[3] Rigney D. Wear 2000;245:1-9.

[4] Cai W, Mabonb J, Bellon P. Wear 2009;267:485-94.

[5] Prasad S, Battaile C, Kotula P. Scripta Mater 2011;64:729-32.

[6] Gahr K-H. Microstructure and wear of materials. Elsevier; 1987.

[7] Hirth J, Rigney D. In: Nabarro F, editor. Dislocations in solids, vol. 6. Amsterdam: Elsevier North Holland; 1983. p. 3-49.

[8] Jang J, Koch C. Scripta Metall Mater 1990;24:1599-604.

[9] Meyers M, Mishra A, Benson D. Prog Mater Sci 2006;51:427556.

[10] Schuh CA, Nieh TG, Yamasaki T. Scripta Mater 2002;46:735-40.

[11] Misra A, Hirth J, Kung H. Philos Mag 2002;82:2935-51.

[12] Nix W, Gao H. J Mech Phys Solids 1998;46:411-25.

[13] Fleck NA, Hutchinson JW. In: Hutchinson JW, Wu JW, editors. Advances in applied mechanics, vol. 33. New York: Academic Press; 1997. p. 296-361.

[14] Greer J, Hosson JD. Prog Mater Sci 2011;56:654-724.

[15] Uchic MD, Shade PA, Dimiduk D. Ann Rev Mater Res 2009;39:361-86.

[16] Greer J, Nix W. Phys Rev B 2006;73:245410.

[17] Volkert C, Lilleodden E. Philos Mag 2006;86:5567-79.

[18] Prasad S, Michael J, Christensen T. Scripta Mater 2003;48:255-60.

[19] Salesky W, Fisher R, Ritchie R, Thomas G. In: Ludema K, editor. Wear of materials. ASME; 1983. p. 434 45.

[20] Hughes D, Dawson D, Korellis J, Weingarten L. J Mater Eng Perf 1994;3:459-75.

[21] Greer JR, Oliver WC, Nix WD. Acta Mater 2005;52:1821-30.

[22] Oliver W, Pharr G. J Mater Res 1992;7:1564-83.

[23] Durst K, Backes B, Franke O, Göken M. Acta Mater 2006;54:2547-55.

[24] Rester M, Motz C, Pippan R. Acta Mater 2007;55:6427-35.

[25] Demir E, Raabe D, Zaafarani N, Zaefferer S. Acta Mater 2009;57:559-69.

[26] Kreuzer H, Pippan R. Acta Mater 2007;55:3229-35.

[27] Pharr GM, Herbert EG, Gao Y. Anu Rev Mater Res 2010;40:271-92.

[28] Kiener D, Pippan R, Motz C, Kreuzer H. Acta Mater 2006;54:2801-11.

[29] Kim J-Y, Kang S-K, Greer J, Kwon D. Acta Mater 2008;56:3338-43.

[30] Backes B, Huang Y, Göken M, Durst K. J Mater Res 2009;24:1197-207.

[31] Uchic M, Dimiduk D, Florando J, Nix W. Science 2004;305:986-9.

[32] Oh S, Legros M, Kiener D, Gruber P, Dehm G. Acta Mater 2007;55:5578.

[33] Shan W, Mishra R, Asif SS, Warren O, Minor A. Nat Mater 2007;7:115-9.

[34] Tang H, Schwarz K, Espinosa H. Phys Rev Lett 2008;100:185503.

[35] Rao S, Dimiduk D, Parthasarathy T, Uchic M, Tang M, Woodward C. Acta Mater 2008;56:3245-59.

[36] Oh SH, Legros M, Kiener D, Dehm G. Nat Mater 2009;8:95-100. 
[37] Motz C, Weygand D, Senger J, Gumbsch P. Acta Mater 2009;57:1744-54.

[38] Liu Z, Liu X, Zhuang Z, You X. Scripta Mater 2009;60:594-7.

[39] Lee S-W, Nix WD. Mater Eng Sci A 2010;527:1903-10.

[40] Weinberger CR, Cai W. Scripta Mater 2011;64:529.

[41] Parthasarathy TA, Rao SI, Dimiduk DM, Uchic MD, Trinkle DR. Scripta Mater 2007;56:313-6.
[42] Nix W, Lee S. Philos Mag 2011;91:1084-96.

[43] Jennings AT, Li J, Greer JR. Acta Mater 2011;59:5627-37.

[44] Bei H, Shim S, Pharr G, George E. Acta Mater 2009;56:4762-70.

[45] Lee S, Han S, Nix W. Acta Mater 2009;57:4404-15.

[46] Jang D, Greer J. Script Mater 2011;64:77-80.

[47] Ghoniem NM, El-Awady JA, Wen M. J Mech Phys Solids 2009;57:32-50 